\title{
Colliding Malignant Lymphoma and Adenocarcinoma of the Stomach: Case Report
}

\author{
Jun Kolke, Tatsuya Ishido, ${ }^{*}$ Noriaki TakiguchI, ${ }^{* *}$ Haruhiko TachI, Junichi TAzaWA, Masanobu \\ Nishimura, Kenichi Kawada and Hideomi Furrwara
}

\begin{abstract}
We report a case of malignant lymphoma presenting as the superior vena cava syndrome. This case is very interesting since both adenocarcinoma mucocellulare scirrhosum, Borrman type IV, and lymphoma were present in the stomach, forming colliding neoplasms. Rather than systemically disseminated, the lymphoma cells were localized in the stomach, the intestine, a few perigastric lymph nodes and in a mediastinal mass, which may have constituted a neoplastic thymus invaded by lymphoma cells from the stomach. The unique occurrence of the histological combination as gastric colliding neoplasms is discussed.
\end{abstract}

(Internal Medicine 31: 86-90, 1992)

Key words: adenocarcinoma mucocellulare, mediastinal mass

\section{Introduction}

Collision tumors are defined as two coexisting tumors which have developed at different sites and have invaded each other in the region of contact during their growth (1). Here, we report a rare case in which advanced adenocarcinoma and malignant lymphoma, simultaneously occurring in the stomach, formed collision tumors.

\section{Case Report}

A 36-yr-old man consulted our department on September 3, 1989, complaining of swelling and pain in his neck. His sister had rheumatoid arthritis and pure red-cell aplasia. The patient had no history of serious illnesses.

About 15 months prior to admission, the patient first noticed a hard tumor of about $1 \mathrm{~cm}$ in diameter in the left antero-inferior region of his neck in the supraclavicular fossa. In May 1989, he noted a swelling in the lower part of his neck, and the swelling was progressive. Near the end of August, transient edema of the lower half of his face occurred when attempting to expectorate sputum. Two days prior to admission, he experienced a brief period of epistaxis and felt dyspneic when he bent forward at the neck. While lying down in the evening of the day of admission the patient experienced a sensation of oppression in his chest, congestion in his head, swelling of his neck and neck pain. There was no history of digestive tract symptoms and the patient was very active and had a good appetite.

On physical examination, the patient was found to be a well-nourished male (ht $166 \mathrm{~cm}$, wt $75 \mathrm{~kg}$ ) who was afebrile, with a heart rate of $114 / \mathrm{min}$ and regular, and a blood pressure of $168 / 90 \mathrm{mHg}$. His bulbar and palpebral conjunctivae were moderately injected, and swelling of both parotid glands was noted. The patient's neck was distended to the manubrial notch. The jugular veins were dilated bilaterally. A hard tumor $(4 \times 2 \mathrm{~cm})$ was found just above the left clavicle. No other superficial lymph nodes were palpable. Examination of the chest, the abdomen and the nervous system revealed no abnormal findings.

Hematologic parameters were as follows: hemoglobin, $15.1 \mathrm{~g} / \mathrm{dl}$; hematocrit, $45.3 \%$; erythrocyte count, $469 \times$ $10^{4} / \mu \mathrm{l}$; leukocyte count, $5,600 / 1$ with $7 \%$ band forms, $74 \%$ segmented neutrophils, $1 \%$ basophils, $1 \%$ monocytes and $17 \%$ lymphocytes. Blood chemistry and serologic findings were almost completely within normal limits. Serum immunoelectrophoresis showed a slight increase in haptoglobin and a slight decrease in IgG.

From Departments of Internal Medicine, ${ }^{*}$ Pathology and **Surgery, Tsuchiura Kyodo Hospital, Tsuchiura

Received for publication September 21, 1990; Accepted for publication April 30, 1991

Reprint requests should be addressed to Jun Koike, MD, Department of Internal Medicine, Hokushin Sogo Hospital, 1-5-63 Nishi, Nakano 383, Japan 


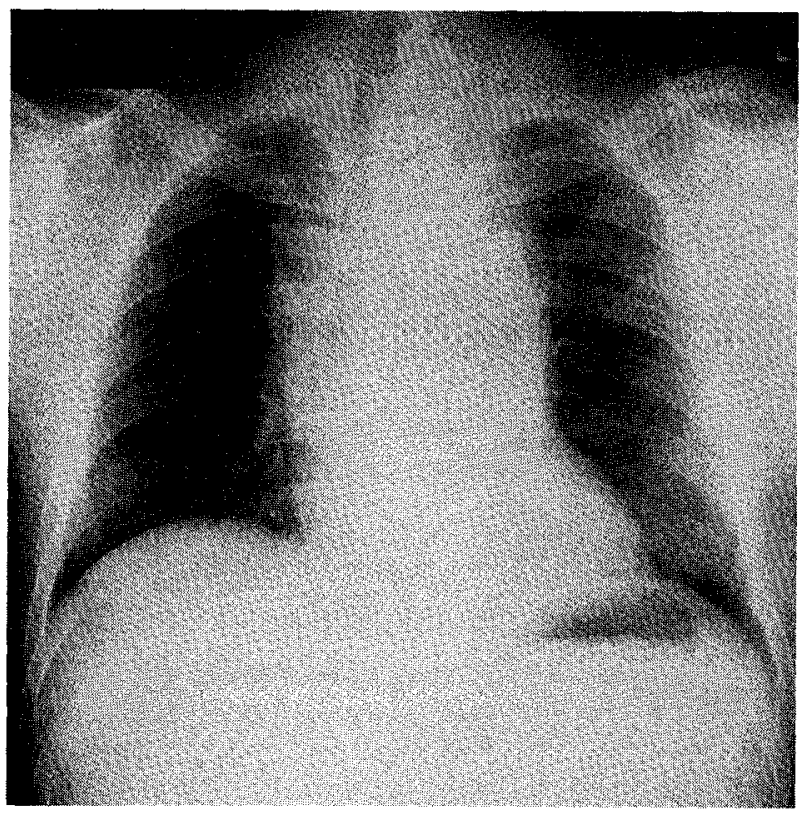

Fig. 1. Chest X-ray (posterior-anterior view). Marked enlargement of the superior mediastinum and great shift of the narrowed trachea to the right.

Serological tumor markers were negative. Since a chest $\mathrm{X}$-ray revealed a large mass in the superior mediastinum, it was apparent that his syptoms were manifestations of suprior vena cava syndrome (Fig. 1). An ultrasonographic examination disclosed a large echolucent area in the superior mediastinum and a $2.5 \times 1.5 \mathrm{~cm}$ echolucent cyst in the left lower neck. Computed tomography scan revealed a large non-enhanced mass located in the anterior, middle, and superior mediastinum which was distributed longitudinally from the level of the sixth thoracic vertebra all the way up to the level of the seventh cervical vertebra (Fig. 2); the mass did not have a distinct border with the trachea which was greatly narrowed and shifted to the right. No pleural invasion was detected and the lung fields seemed to be intact. No invasion to the aorta or pulmonary artery was apparent even though there was an obvious backward shift of the aorta. Bronchoscopy disclosed disapperance of the cricoid ring from the mucosal surface of the trachea and an injected and edematous mucosal surface, suggesting invasion of the mass into the submucosa of the trachea. Fine-needle aspiration cytology of the superior mediastinal mass suggested malignant lymphoma or malignant thymoma.

Upper gastrointestinal endoscopy revealed a Borrman III-like lesion on the posterior wall of the body of the stomach and a few submucosal elevated masses on the anterior wall of the body. Biopsy specimens of the former showed the histological picture of adenocarcinoma mucocellulare, however, the latter showed malignant lymphoma. Duodenoscopy revealed submucosal tumors in the wall of the upper portion of the duodenum. The

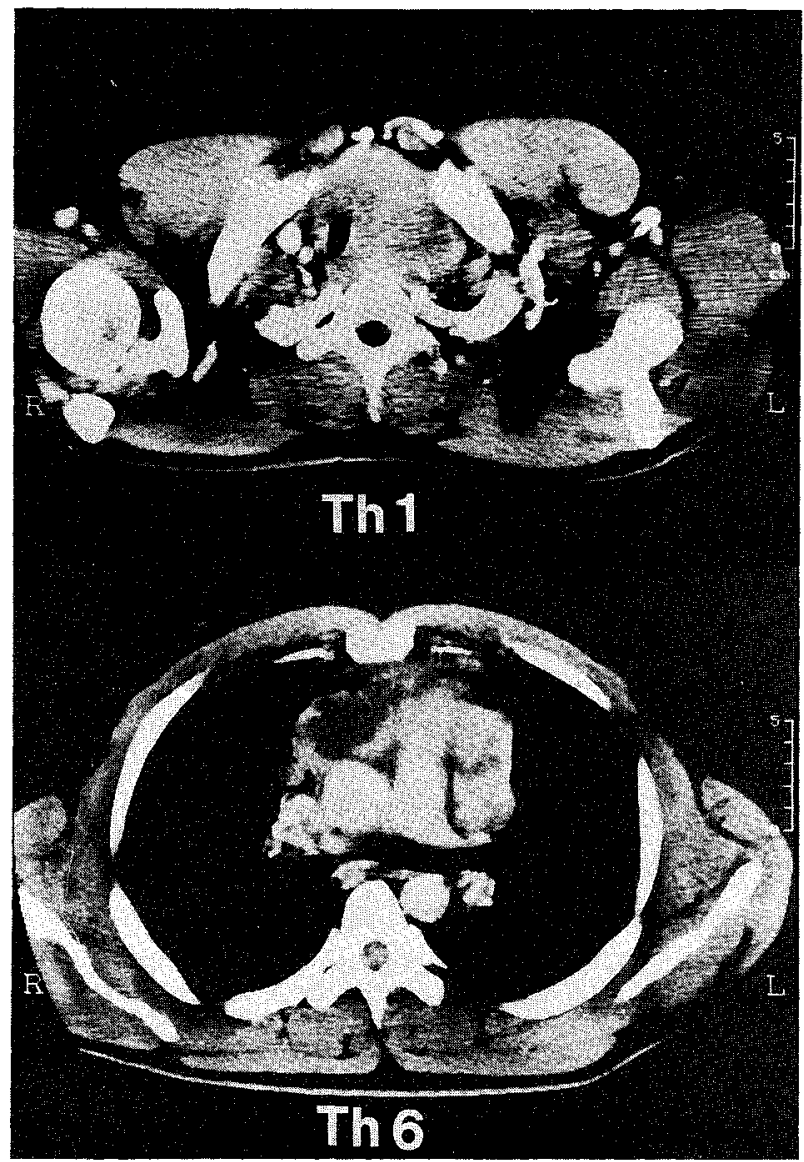

Fig. 2. Computed tomograph of the mediastinum. The upper half is located at the level of the first thoracic vertebra and the lower half, the sixth thoracic vertebra.

esophagus exhibited no obvious submucosal lesions or stenosis. No lymphoma cells were detected in the bone marrow. Ultrasonographic studies and a CT scan of the abdomen failed to reveal any swelling of intra-abdominal lymph nodes, masses in the liver, swelling of the spleen or any tumors in the pancreas.

Surgical resection of the stomach was planned for treatment of adenocarcinoma, since no metastasis was found elsewhere. Prior to surgery, the mediastinum was irradiated in order to shrink the mediastinal mass, and to straighten the trachea, to facilitate intratracheal intubation for the operation. The tumor was very radiationsensitive; a total dose of $1,600 \mathrm{rad}(2 \mathrm{~Gy} \times 8$ days) was adminsterred. This was followed by total gastrectomy, splenectomy and partial pancreotectomy on September 20, 1989. During surgery, flared and hard tumors were found on the wall of the duodenum. Hard tumors were also palpated in the jejunum which were gradually smaller toward the anal end.

After surgery, standard chemotherapy (CHOP) was administered twice to treat the residual malignant lymphoma, but the patient failed to respond to therapy and died on January 20,1990, as a result of systemic infiltration by of lymphoma cells. 


\section{Pathology}

Gross examination of the surgically resected stomach revealed some polypoid lesions, swollen gastric folds and erosion of the mucosal surface (Fig. 3). Microscopic examination disclosed malignant Lymphoma of the stomach which was a diffuse, mediumsize type according to the LSG classification (Fig. 4a); occasional cleaved cells were seen, suggesting that they might be of the B cell type. Lymphoma cells were widely distributed throughout the stomach, from the antrum to the fornix; they had spread into the duodenum and had infiltrated into the serosa. There was an irregular, ulcerative, $1 \mathrm{~cm} \times$ $1 \mathrm{~cm}$ lesion on the posterior wall of the body of the stomach, close to the minor curvature. Adenocarcinoma mucocellulare scirrhosum, pre-linitis plastica type (Fig. $4 b$ ) was located around the ulcerative lesion. The interrelation and distribution of the two neoplasms is shown schematically in Fig. 5. The two lesions were very intermingled and both neoplastic cells were admixed in the overlapping region (Fig. 4c, d). Only lymphoma

Fig. 3. Gross findings of the resected stomach showing an ulcerative lesion (arrow) and adenocarcinoma mucocellulare which is distributed submucosally (bar $=5 \mathrm{~cm}$ ).

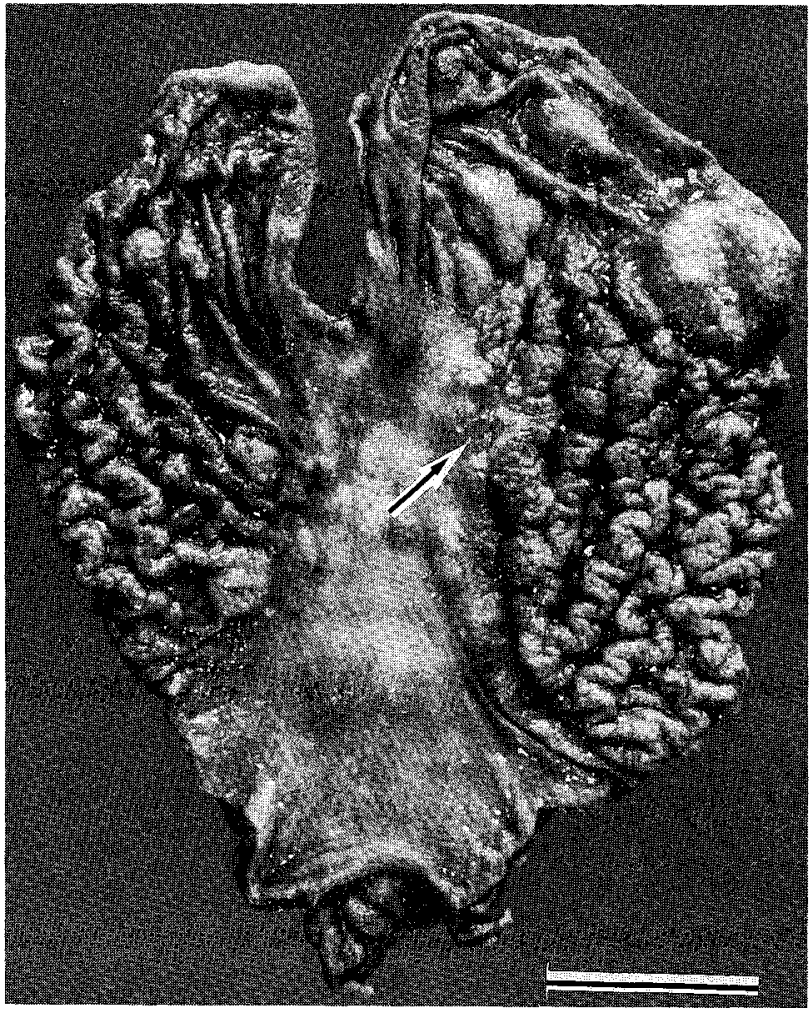

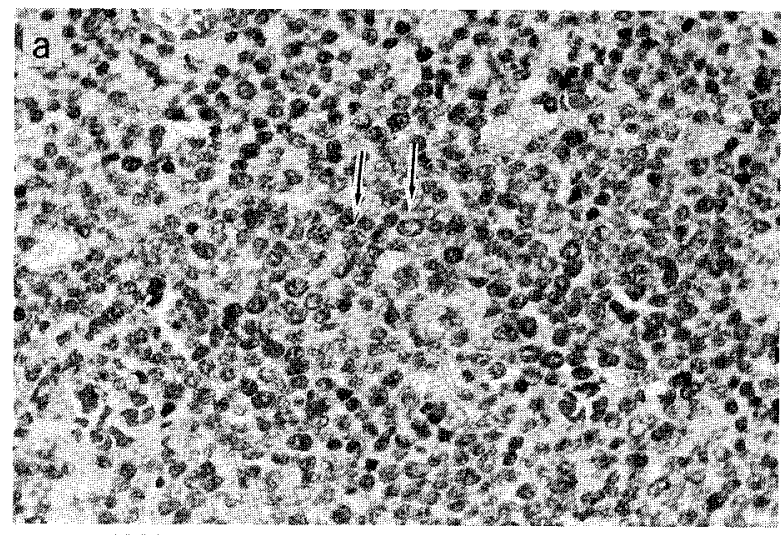

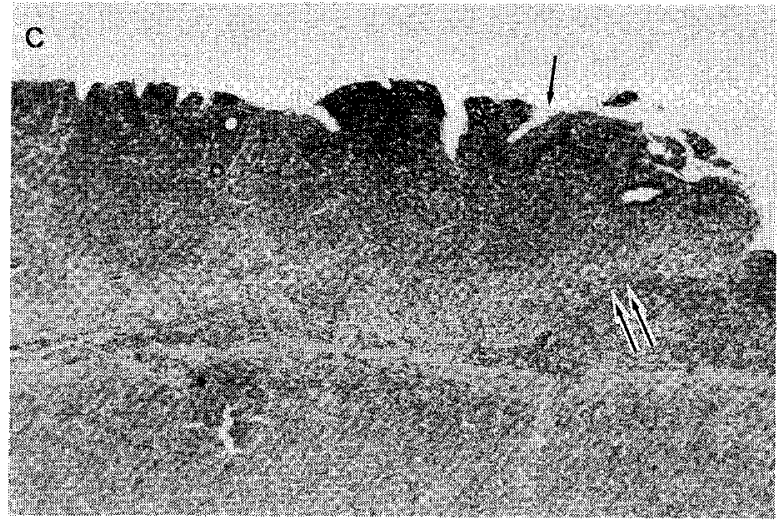

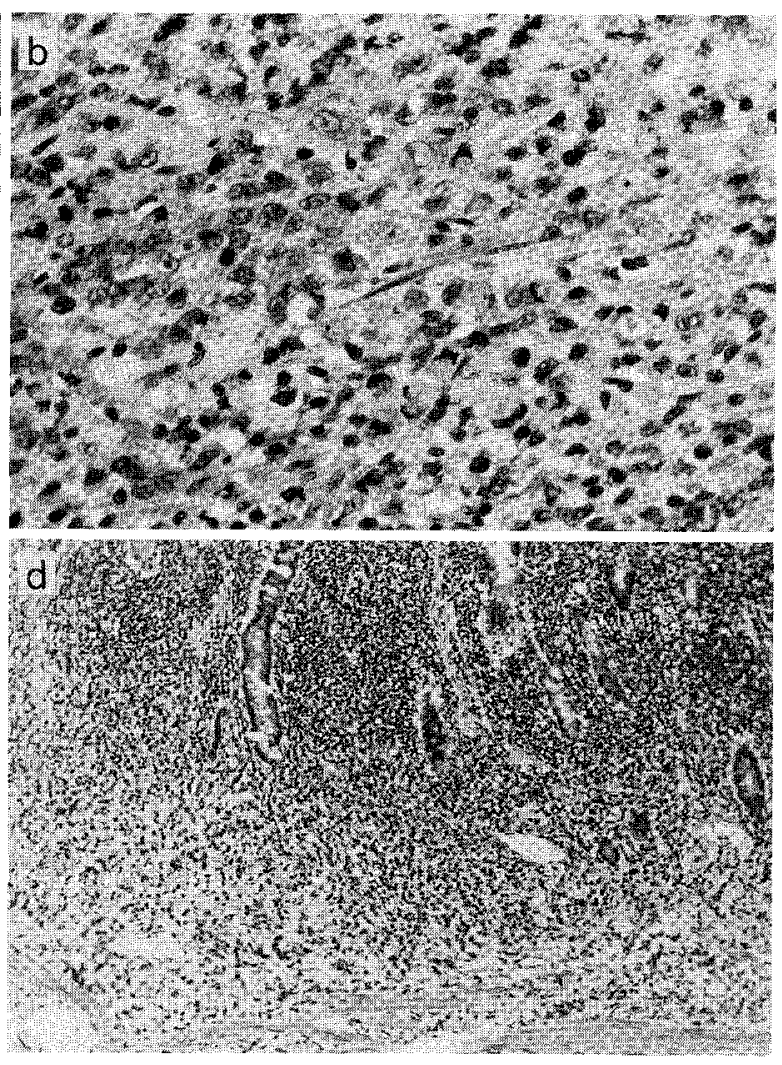

Fig. 4. a) High power view of the malignant lymphoma of the stomach. Arrows indicate cleaved cells $(\mathrm{H}$ and $\mathrm{E}, \times 226)$. b) High power view of the adenocarcinoma mucocellulare of the stomach. Mucin laden cells are easily detectable, but there is no gland formation $(H$ and $E, \times 226)$. c) Collision of the lymphoma and the carcinoma. Single arrows: region of the lymphoma; double arrows: region of the carcinoma $(\mathrm{H}$ and $\mathrm{E}, \times 11)$. d) Collision findings. No clear boundary is formed; there is continuous intermingling $(\mathrm{H}$ and $\mathrm{E}, \times 44)$. 


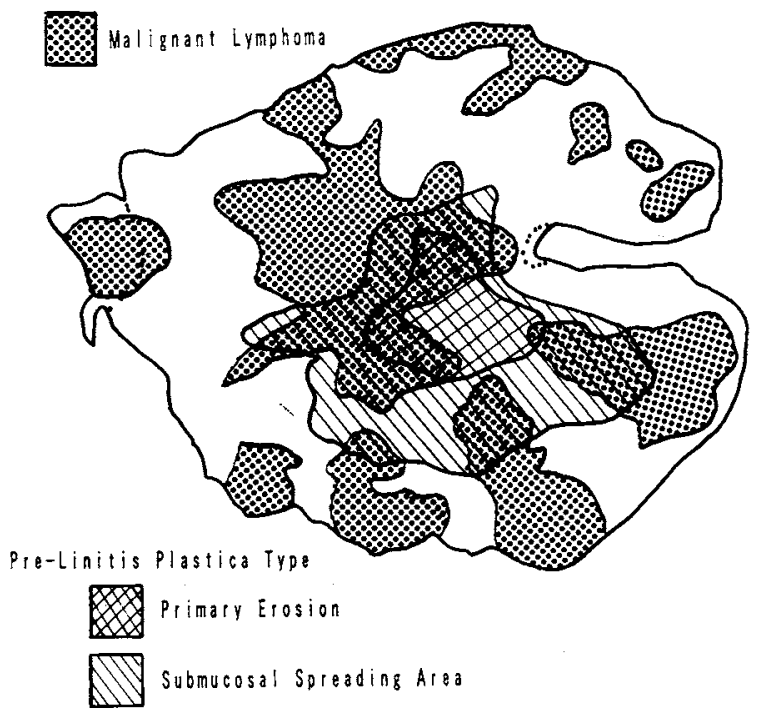

Fig. 5. Schematic representation of the gastric neoplasms. The dotted areas represent the distribution of the lymphoma. The multicentric nature of the lymphoma in the stomach can be seen. The single-hatched and cross-hatched areas show the location of the carcinoma.

cells were found in the four perigastric lymph nodes; no adenocarcinoma cells were found in any of the lymph nodes. The resected parts of the pancreas and the spleen displayed no signs of malignancy.

The mass removed from the anterior superior mediastinum at autopsy was soft and elastic, and $14 \times 7 \mathrm{~cm}$ in size (Fig. 6). It was located precisely where normal thymic tissue would have been expected. The orad end was thin and located approximately retrosternally. From there it extended longitudinally between both pleura and the pericardium, becoming increasingly thick. The mass was adhered to the surrounding pericardium and pleura but did not directly invade the parenchyma of the lung or heart. Since no normal thymus tissue was detected, the mass was believed to represent a neoplastic thymus. The histology of the mass is shown in Fig. 7 together with one of the mesenteric lymph nodes. These were identified to be the diffuse mediumsize type according to the LSG classification, the same type as the lymphoma in the stomach. Hence, they were considered to have the same neoplastic cell origin. No surface monoclonal immunogloblins, MB1, or MT1, were detected on any paraffin sections of the stomach, the lymph node or the mediastinal mass as markers. Electron microscopy of the lymph node also revealed malignant lymphoma of the mediumsize type. Lymphoma cells had infiltrated the small intestine, colon, and pancreas. The peripancreatic lymph nodes had formed a few tumors ( $2 \mathrm{~cm}$ maximum diameter) which had not been observed at the time of surgery. Such tumors were also found in the portal area of the liver, along the bronchi and the pulmonary artery. No adenocarcinoma cells were detected at autopsy despite the poor differentiation and advanced nature of the

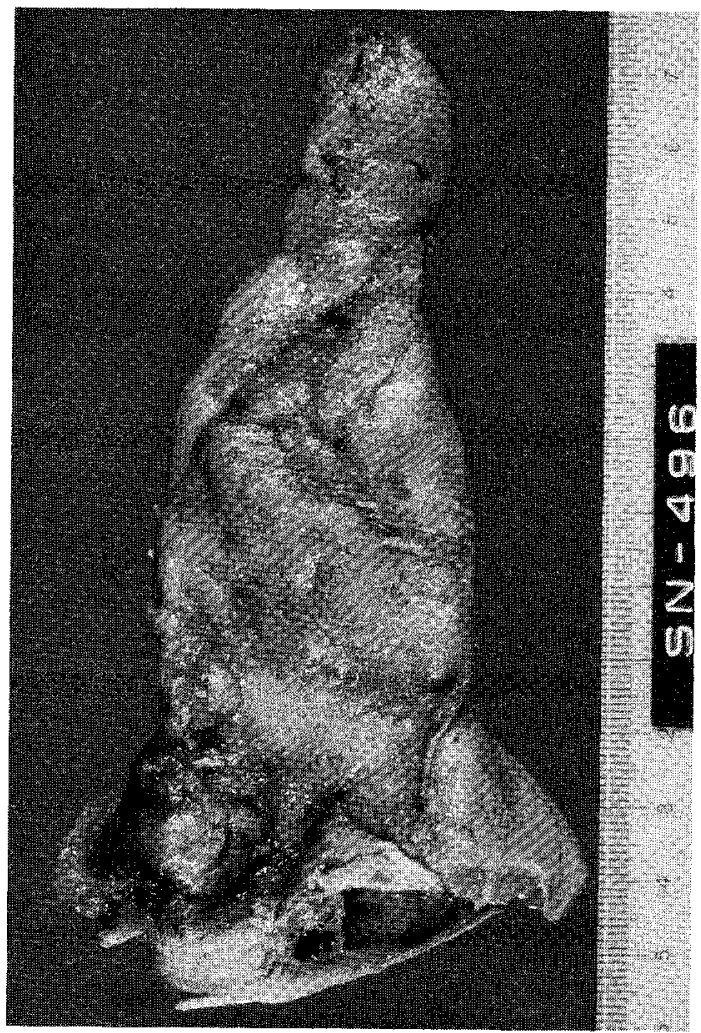

Fig. 6. Gross findings of the mediastinal mass, consisting of fibrous tissue and fat (scale $=1 \mathrm{~cm}$ ).
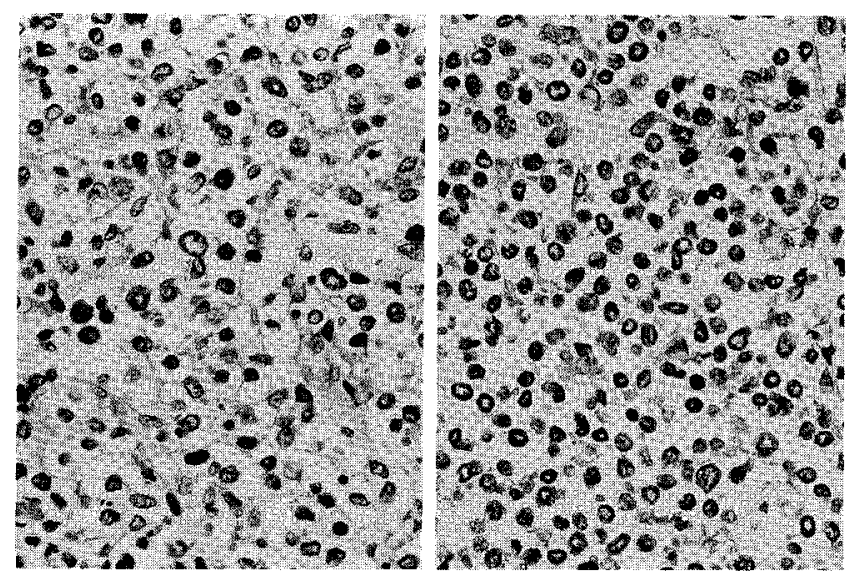

Fig. 7. High power view of the mediastinal mass (left half) and the mesenteric lymph node (right half) $(\mathrm{H}$ and $\mathrm{E}, \times 226)$.

carcinoma.

\section{Discussion}

It seems that the mediastinal mass originated in the thymus, based on the macroscopic findings described above, although no histological evidence, such as thymic Hassall-Henle bodies were observed. It is of interest to determine whether in this case the thymus was the primary site of the patient's disease or a site invaded by 
lymphoma cells from the upper gastrointestinal tract. It had often been observed that lymphoma cells from the stomach infiltrate a Virchow's lymph node. So if we assume the presence of residual thymic tissue which developed in the patient's neck during the embryonal period, the lymphoma may have invaded the thymus. On the other hand, no esophageal lesions or swelling of lymph nodes in the posterior mediastinum were observed at autopsy, thus it seems rather unfeasible that lymphoma cells from the thymus lymphogenously invaded the stomach retrogradely via the lymphatic duct in the posterior mediastinum. Hence, the thymus seems to have been the site of invasion by the lymphoma in the stomach rather than the primary site. To the best of our knowledge, no cases of simultaneous thymic lymphoma and gastric lymphoma have been reported yet with the exception of the disseminated type; this case might be considered a case in which the lymphoma cells were mainly localized in the stomach, the small intestine and the thymus, at least when the patient was admitted. Further, there seems to be no reports of the thymus invaded by lymphoma cells from the stomach or other organs [for a review of thymic neoplasia, see Gerald et al (2)].

The simultaneous presence of multiple carcinomas in the stomach is not an unusual phenomenon. A recent Japanese study of early gastric cancer reported the incidence of multiple early carcinomas as $8 \%$ ( 73 out of 900 cases) (3). The coexistence of malignant lymphoma and adenocarcinoma $(4,6-10)$ is rare, however. Noda et al (5) reported that the incidence of coexisting primary malignant lymphoma and adenocarcinoma of the stomach is only $0.08 \%$ ( 2 of 2,438 cases). According to Kasahara et al (4), less than 20 cases have been reported in the English and German literature and 35 cases in the Japanese literature. Among these Japanese cases, undifferentiated carcinomas were found in only four out of 27 histologically described adenocarcinomas. As far as we know, the coexistence of malignant lymphoma and Borrman type IV adenocarcinoma mucocellulare has never been reported. Only one case of adenocarcinoma mucocellulare has been described in the Japanese literature as an early cancer (6). Kane et al (7) have stated that the lymphomas in simultaneous carcinoma and lymphoma of the stomach are predominantly histiocytic. The lymphoma in the present case, however, was not histiocytic. Therefore, regarding collision tumors of gastric malignant lymphoma and cancer, such a histological combination is unique considering both the adenocarcinoma and the malignant lymphoma. The priority of one neoplasm over the other could not be determined. Finally, it should be noted that the coexistence of these two tumors had been positively diagnosed on the basis of endoscopic examination and biopsy prior to surgical resection of the stomach.

Acknowledgments: The authors are greatful to Dr. K. Tomita and Dr. H. Nonoguchi for their careful reading of the manuscript and useful suggestions, and to thank Ms. E. Ishii for her assistance in drawing a figure.

\section{References}

1) Meyer R. Beitrag zur Verstandigung über die Namendebung in der Geschwulstlehre. Zentralbl Allg Pathol 30: 291, 1919.

2) Gerald D, Levine $\mathrm{MB}, \mathrm{Ch} \mathrm{B}$, et al. Thymic hyperplasia and neoplasia: A review of current concept. Prog Hum Pathol 9: 495, 1978.

3) Hirota T, Itahashi M, Suzuki K, et al. Clinicopathological study of minute and small early gastric cancer. in: Pathology Annual 1980, Sommers SC, Rosen PP, Eds. Appleton-Century-Croft, East Norwalk, Conn, 1980, part 2, p.1.

4) Kasahara $Y$, Takemoto M, Morishita A, et al. Coexisting adenocarcinoma and malignant lymphoma of the stomach. Am J Gastroenterol 83: 190, 1988.

5) Noda $T$, Akaishi $H$, Matsueda $S$, et al. Collision of malignant lymphoma and multiple early adenocarcinomas of the stomach. Arch Pathol Lab Med 115: 419, 1989.

6) Matsumoto T, Matsumoto A, Sekine M, Sugiura Y. Coexistence of malignant lymphoma and early carcinoma in the stomach. Prog Dig Endosc 20: 269, 1982 (in Japanese).

7) Kane EP, Weingarten LA, Payson BA, et al. Synchronous ulcerating adenocarcinoma and malignant lymphoma of the stomach. Am J Gastroenterol 77: 461, 1982.

8) Manier JW, Reyes CN. Collision tumor of the stomach. Gastroenterology 67: 1011, 1974.

9) Planker M, Fischer JT, Peter U, et al. Synchronous double Primary malignant lymphoma of low grade malignancy and early cancer (collision tumor) of the stomach. Hepatogasroenterology 31: $144,1984$.

10) Czerniak A, Lotan G, Engelberg IS, et al. The simultaneous coexistence of adenocarcinoma and primary malignant lymphoma. J Surg Oncol 30: 42, 1985. 